\title{
Self-Adaptive Anisotropic Image Enhancement Algorithm Based on Local Variance
}

\author{
Yan Chen ${ }^{1,2}$, Yunjiao Bai ${ }^{1}$, Quan Zhang ${ }^{1}$, Yanling Wang ${ }^{1}$ and Zhiguo Gui ${ }^{1, *}$ \\ ${ }^{I}$ School of Information and Communication Engineering, North University of China, Taiyuan 030051, China \\ ${ }^{2}$ School of Engineering, Southern Illinois University, Edwardsville, Illinois, 62026, USA
}

Received 14 February 2017; Accepted 2 June 2017

\begin{abstract}
When an image is enhanced using the traditional Laplacian enhancement model, the enhancement effect is evident but the overshoot phenomenon occurs simultaneously as the neighborhood and weight increase. To solve the contradiction between edge preservation and noise suppression during the image enhancement process, this study proposed an improved partial differential equation image enhancement algorithm. The algorithm combined the neighborhood features of digital images, mined the relationship between the local variance and the detail information of images, and classified the image noise and detail information with local variance. On this basis, the periodic change features of the trigonometric function were used to construct anisotropic diffusion coefficients, the numerical solution method of the partial differential equation was used to calculate the numerical solution of the proposed algorithm, and simulation experiment was implemented to analyze the influence of the algorithm model parameters on enhancement effect. Lastly, the anisotropic enhancement algorithm proposed in this study was validated through comparison with the traditional Laplace algorithm. Results indicate that tangential direction parameter and the number of iterations exert influence on the image enhancement effects. That is, when tangential direction parameter is set as 0.5 and the iterative operation is conducted 5 times, the algorithm smoothens the noise and enhances the image details. When the proposed algorithm is used to process low-contrast industrial image, the signal-to-noise ratio of the enhanced image is increased by $8 \mathrm{db}$ and the mean squared error is reduced by 55\% compared with the enhancement effect of the traditional Laplace enhancement method. In terms of subjective visual effect, the noise of the enhanced image is filtered, whereas detailed information (e.g., numerous weak edges) is reserved. Hence, subjective and objective evaluations reflected that the proposed algorithm can effectively enhance image details and avoid over-enhancement. Moreover, the proposed algorithm is of favorable adaptability and certain reference value to low-contrast image enhancement.
\end{abstract}

Keywords: Image Enhancement, Anisotropic Diffusion, Laplace

\section{Introduction}

Digital image is influenced by illumination or all types of noise sources during the imaging process; thus, the generated image has blurred edge and reduced feature information. Accordingly, image smoothing should be performed prior to the follow-up processing. Gaussian filtering is the most common image filtering method among the traditional image smoothing methods. However, Gaussian filtering belongs to lowpass filtering, which can process the edge and non-edge of an image in the same filtering manner, thereby blurring the image features and resulting in feature loss. Hence, scholars have been developing a diffusion method that can differentiate the feature information points from the noise points of images. This method can adopt different filtering processes for boundary and flat regions. In recent years, a few scholars have studied image processing methods based on partial differential equation [1]. In particular, integer-order partial differential is used by image enhancement technology based on integer order partial differential to detect image gradient

\footnotetext{
*E-mail address: nucchenyan@126.com

ISSN: 1791-2377 @ 2017 Eastern Macedonia and Thrace Institute of Technology. All rights reserved.

doi:10.25103/jestr.103.09
}

value. Moreover, the enhancement processing of an image with edge sharpening is realized through differential filtering. The Robert operator is a gradient operator based on firstorder differential. The algorithm has favorable lift effect in edges but it is sensitive to noise [2]; thus, it cannot enhance the texture region. The Prewitt operator [3], which is a firstorder average operator, has certain smoothing function on image noise. Accordingly, the edge of the processed image is thick and bright, but the image texture is suppressed and there is a pseudo edge. The Sobel operator is improved based on the Prewitt operator, the pixel is weighted by pixel position. The weight is inversely proportional to the distance from the center point of the pixel. Hence, the enhanced image has substantially clear and bright edges. However, the Sobel operator [4] inhibits texture details; thus, it still needs improvement. The Laplace operator is an image-sharpening operator based on second-order differential. The Laplace equation is a classical partial differential enhancement method of images. When image enhancement is performed using the Laplace isotropic operator, given that highfrequency components are excessively enhanced, the image presents a considerably cluttered effect called the "explosion" phenomenon [5]. The detection of the image edges using the Gaussian Laplace operator [6] can reflect the visual characteristics of the human eyes. In practical application, noise has immense influence on an image 
because the scale factor cannot make self-adaptive adjustment.

Based on the above background, this study presented a self-adaptive diffused anisotropic image enhancement method, which could deal with image noise and edge, enhance image detail information and improve image contrast.

\section{State of the art}

At present, image enhancement methods are mainly divided into the spatial domain and transform domain methods. The spatial domain method directly processes pixel points, and the representative algorithms include the histogram method, the gray-scale transform method, the Retinex method, and other enhancement algorithms. The histogram method is one of the simplest and most effective methods, and uniformly distributes pixel points that concentrate in narrow gray-scale interval inside the original image based on gray-scale statistical characteristics. The advantages of histogram method lie in simple but high-speed algorithm that can automatically enhance images. However, it is sensitive to noise, with easy detail loss and serious distortion occurring in a few regions. HAN [7] proposed an improved histogram equalization algorithm that could effectively solve the problems of over-combination of gray scales and easily detail loss of traditional histogram method. However, the color loss phenomenon would easily happen to the color images. The gray-scale transform method maps relatively centralized low-gray value in the input image into the output gray value in relatively wide interval. The commonly used mapping methods are the logarithmic and the parabolic transformations. This method is of high-processing speed, but parameter adjustment cannot be self-adaptively confirmed based on the image features. Consequently, the supersaturation phenomenon will easily happen in a few image regions. ZHOU et al. [8] proposed a method that simultaneously enhances global brightness and local contrast. This algorithm effectively solved the self-adaption problem of the algorithm in gray-scale transformation; however, the effect of this method on images with less brightness still needs improvement. The Retinex algorithm, which is an image enhancement method of light compensation, reaches an enhancement effect based on the color correction of the gray-scale assumption and can simultaneously realize the enhancement of the global and local contrasts of the image. Through the simulation of the human vision system, the Retinex algorithm obtains continuous development. Given that the essence of the Retinex algorithm is based on grayscale assumption, uniform color region violates gray-scale assumption theory. Thus, the image color after processing is unsaturated and becomes gray tone with substantial color distortion problem. XIAO et al. [9] proposed an improved multi-scale Retinex algorithm with color restoration. This algorithm could effectively solve such problems as color distortion and noise amplification. However, this algorithm is highly complex; thus, it cannot be used in real-time processing.

The transform domain method transforms an image into another spatial domain for processing. The more relatively classical transform domain method is the wavelet transform method. The wavelet coefficients are acquired by doing wavelet transform of an image. Thereafter, the coefficients of the different frequencies within the image are multiplied by the different coefficients to enhance or inhibit the effects of a few components. Image processing technology [10] based on wavelet transform has drawn the considerable attention of scholars because wavelet transform manifests the time-domain features of an image and embodies its frequency-domain features. That is, this technology extracts image edges and the overall structure. This characteristic agrees with the low illumination image enhancement technique. However, this method primarily needs to define wavelet basis; thus, the application of the algorithm is limited.

Since Perona and Malik proposed the anisotropic diffusion model (i.e. Perona-Malik (P-M) model) [11] in 1990 , partial differential equation has developed rapidly in the field of image processing. When the partial differential equation is used to enhance tan image, this equation can directly process significant geometric features, such as gradient, tangential line, and level set, and can effectively simulate the diffusion characteristics of the human vision. In addition, the numerical analysis method of partial differential equation is accurate and stable. Consequently, the advantages of the partial differential equation have drawn substantial attention from a group of local and international experts. Thereafter, the improved denoising models have been developed in succession. Catte proposed a Gaussian regularization model [12], which yielded favorable effects when applied to edge detection and image restoration The variable index model proposed by Guo [13] could selfadaptively control diffusion pattern. P-M diffusion and Gaussian smoothing were conducted based on different image features. Compared with the traditional $\mathrm{P}-\mathrm{M}$ model, the new model effectively extracted image edge features. Ratner used the telegram diffusion equation [14] to substantially reserve the details; hence, it is extensively applied in image processing. Accordingly, You and Kaveh proposed a fourth-order partial differential equation [15] and Bai and Feng introduced a fractional-order partial differential equation [16], thereby enabling the further development of the partial differential equation obtained in the aspect of image denoising. Image enhancement technology based on partial differential equation is an important branch of image processing. Moreover, the most typical one is inverse heat conduction equation, namely the Laplace image enhancement algorithm. This equation conducts isotropic enhancement of the image. Moreover, it can substantially enhance image features but cannot inhibit noise and will easily generate the overshoot phenomenon. An improved algorithm proposed by Wang based on PDE(Partial Differential Equation)solved the enhancement problem of nonlinear dim target [17]. The dim target image was enhanced by the algorithm by importing the proper gradient threshold values and sharpening intensity parameters. However, the algorithm still had non-ideal enhancement effect because the diffusion direction had incomplete self-adaptability.

In summary, image enhancement technology has obtained certain achievements. However, existing algorithms encounter numerous problems, such as easy detail loss, color distortion and high algorithm complexity. Moreover, lowcontrast image enhancement technology has yet to guarantee the performance of a visual system under low-light environment. This study used the analysis of the partial differential equation as basis to propose a method that can effectively improve image contrast.

The remainder of this study is organized as follows. Section 2 expounds on the main research conditions of the digital image enhancement algorithms. Section 3 proposes 
Yan Chen, Yunjiao Bai, Quan Zhang, Yanling Wang and Zhiguo Gui/

Journal of Engineering Science and Technology Review 10 (3) (2017) 64-72

the improved algorithm, analyzes the problems that should be solved in image enhancement, and particularly proposes a self-adaptive anisotropic enhancement algorithm that combines local variance and trigonometric function. Section 4 analyzes the influence of several important parameters on the proposed algorithm, as well as compares the proposed algorithm with the traditional Laplace algorithm. Section 5 summarizes the conclusions.

\section{Methodology}

\subsection{Laplace image enhancement algorithm}

The Laplace image enhancement algorithm is a commonly used image detail enhancement method. Dennis Gabor proposed that inverting the time direction of the heat conduction model could enhance high-frequency components. The temporal evolution pattern of Gabor is as follows:

$$
\left\{\begin{array}{l}
\frac{\partial f(x, y, t)}{\partial t}=-\Delta f(x, y, t), t>0 \\
f(x, y, 0)=f(x, y)
\end{array}\right.
$$

For the convenience of solving this equation, the skills of solving standard heat conduction equation can be used: firstorder Taylor series expansion. The specific process is as follows: spatial variables $x$ and $y$ are fixed, $f(x, y, t)$ is expanded based on the Taylor series nearby $t=0$ about $t$. The following equation can be obtained:

$$
\begin{aligned}
f(x, y, t) & \approx f(x, y, 0)+t\left(\frac{\partial f(x, y, t)}{\partial t}\right)_{t=0} \\
& =f(x, y)-t \Delta f(x, y)
\end{aligned}
$$

This equation is called Laplace algorithm and $\Delta$ is the Laplace operator. The $\Delta$ operator is isotropic and can be decomposed based on the gradient direction $n$ and tangential direction $s$ orthogonal to $n$ :

$$
\Delta=\frac{\partial^{2}}{\partial n^{2}}+\frac{\partial^{2}}{\partial s^{2}}
$$

Hence, the standard Laplace algorithm can be rewritten into the following form:

$$
f_{t}(x, y)=f(x, y)-t\left(\frac{\partial^{2} f}{\partial n^{2}}+\frac{\partial^{2} f}{\partial s^{2}}\right)
$$

In practical engineering, the Laplace operator is considerably convenient. One of masks in the following three forms is often adopted.

\begin{tabular}{|c|c|c|}
\hline 0 & 1 & 0 \\
\hline 1 & -4 & 1 \\
\hline 0 & 1 & 0 \\
\hline
\end{tabular}

(a)

\begin{tabular}{|c|c|c|}
\hline 1 & 1 & 1 \\
\hline 1 & -8 & 1 \\
\hline 1 & 1 & 1 \\
\hline
\end{tabular}

(b)

\begin{tabular}{|c|c|c|}
\hline 1 & 4 & 1 \\
\hline 4 & -20 & 4 \\
\hline 1 & 4 & 1 \\
\hline
\end{tabular}

(c)
Fig. 1. Laplace Operator Mask

For the convenience of analyzing the Laplace enhancement algorithm, the preceding three masks from left to right are labeled the mask (a),(b)and (c)(see Fig. 1). The different masks will obtain images with different enhancement effects. To specify the enhancement effects of the different masks, three masks are used in this study to process the image of peppers. Figure 2 is the resulting graph of processing through different masks. From the detailed drawing extraction, it can be seen that different the Laplace operators are used to extract the detail information of the image, and the feature extraction is more obvious with the increase of template weight coefficient. Compared with the original image, the image enhanced using the Laplace algorithm preserves the edge detail features in the image while amplifying background noise. The weight coefficient of the third mask is the maximum. Compared with the first two masks, the third mask can substantially extract the detailed features; however, the image noise becomes considerably serious with severe overshoot phenomenon.

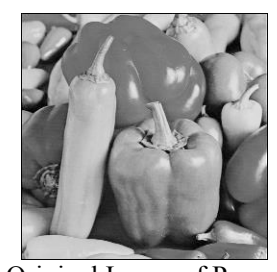

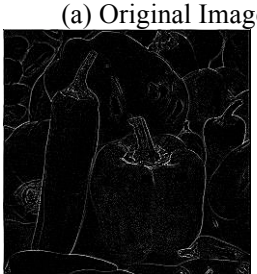

(b) Detailed Extracted By Mask(a)

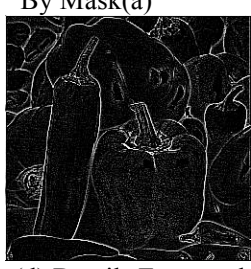

(d) Details Extracted

by Mask(b)

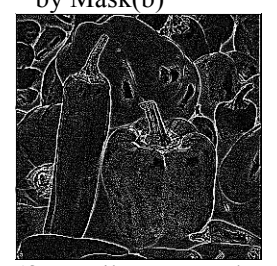

(f) Details Extracted by Mask(c)

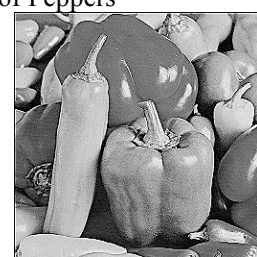

(c) Image Enhanced through Mask(a)

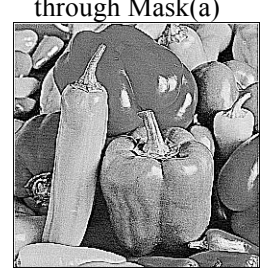

(e) Image Enhanced

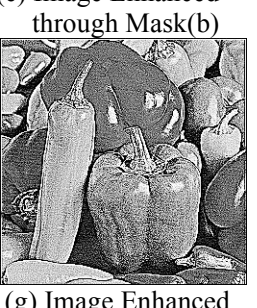

(g) Image Enhanced through Mask (c)
Fig. 2. Results of the Comparison of the Image Enhancement of Peppers Using the Three Masks

The Laplace operator is used to perform the isotropic enhancement of the image. Accordingly, the algorithm can enhance the details of the image but it also amplifies noise. From four neighborhoods to eight neighborhoods and as weights increases, the enhancement effect becomes substantially evident. However, severe overshoot phenomenon appeares in the boundary with extreme noise pollution.

\subsection{Self-adaptive anisotropic enhancement algorithm based on local variance}

\subsubsection{Basic principles of the algorithm}

Given that the traditional Laplace image enhancement belongs to the isotropic enhancement algorithm, this algorithm is sensitive to noise, as well as amplifies noise 
Yan Chen, Yunjiao Bai, Quan Zhang, Yanling Wang and Zhiguo Gui/

Journal of Engineering Science and Technology Review 10 (3) (2017) 64-72

while enhancing the image details. Moreover, the highfrequency components are over-enhanced and the image presents considerably cluttered effect. In this study, the tangential and normal diffusion coefficients are selfadaptively adjusted as well. Thereafter, self-adaptive anisotropic enhancement algorithm based on the local variance is proposed as follows:

$f_{t}(x, y)=f(x, y)+t\left(\alpha \cdot \frac{\partial^{2} f}{\partial n^{2}}+\beta \cdot \frac{\partial^{2} f}{\partial s^{2}}\right)$

where $\alpha$ is the function of the local variance of the image and $\beta$ is a positive constant. The core idea of the algorithm is to use the method that combines forward diffusion and reverse diffusion to enhance the radial image.

Given that the gray-scale variance can effectively differentiate image details from noise-containing background [18], the local variance is imported to classify image features and construct the self-adaptive diffusion coefficient. The local variance $\sigma^{2}(x, y)$ of the image within a $3 \times 3$ domain at point $(x, y)$ is defined as follows:

$$
\sigma^{2}(x, y)=\frac{1}{9} \sum_{i=-1}^{1} \sum_{j=-1}^{1}(f(x+i, y+j)-\bar{f}(x, y))^{2}
$$

where $\bar{f}(x, y)$ is the gray average of the $3 \times 3$ window neighborhood. For the convenience of parameter selection, the local variance is normalized to $0-255$ in this study, particularly as follows:

$\sigma_{N}^{2}(x, y)=\frac{\sigma^{2}(x, y)-M i n \sigma^{2}}{M a x \sigma^{2}-M i n \sigma^{2}} \cdot 255$

where $M i n \sigma^{2}$ and $M a x \sigma^{2}$ are the minimum and maximum values, respectively, of the local variance of the image.

Therefore, the change characteristics of the trigonometric function curve are used to construct the diffusion coefficients. $T$ is set as the variance threshold value and $2 \times T$ and $4 \times(255-T)$ are the cycles to design the cosine function. The function $\alpha\left(\sigma^{2}\right)$ regarding the local variance is constructed to control the normal diffusion coefficients as follows:

$\alpha\left(\sigma^{2}\right)=\left\{\begin{array}{cc}\cos \left(\frac{\pi}{T} \times \sigma^{2}\right) & \sigma^{2} \leq T \\ \cos \left(\frac{\pi}{2 \times(255-T)} \times\left(\sigma^{2}-3 \times T+510\right)\right) & \sigma^{2}>T\end{array}\right.$

Fig. 3 shows the change curves of function $\alpha\left(\sigma^{2}\right)$ at $T_{1}=50$ and $T_{2}=100$. When the local variance is within $0-T / 2, \alpha\left(\sigma^{2}\right)$ is a positive number and equation (5) is a forward diffusion model. When the local variance is above $T / 2, \alpha\left(\sigma^{2}\right)$ is a negative number and equation (5) is a reverse diffusion model. Moreover, as the variance threshold value $T$ is reduced, the region of positive $\alpha\left(\sigma^{2}\right)$ decreases. So that, the diffusion coefficient of $\alpha\left(\sigma^{2}\right)$ as the normal direction is more conducive to detail enhancement and detail protection.

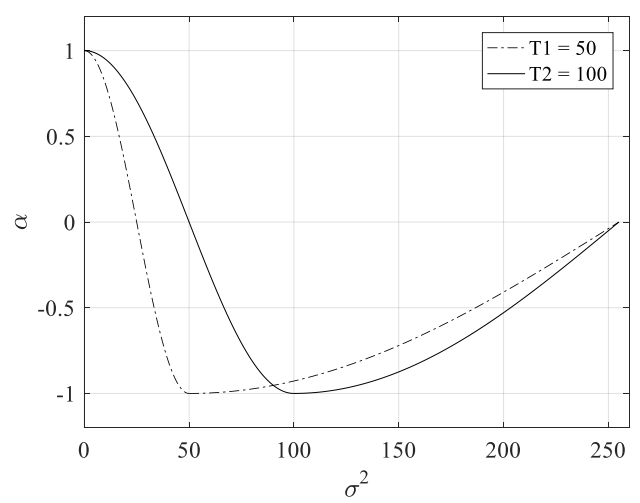

Fig. 3. Change Curves of Function

The preceding analysis indicates that $T$ is gradually reduced as the iterations proceed. Moreover, the selfadaptive threshold value $T$ is used and defined as follows:

$$
T=T_{0} \cdot e^{-n}
$$

where $n$ is the number of iterations and $T_{0}$ is a positive constant. Fig. 4 shows that the change curve of $T$ as the number of iterations increases. Evidently, when the number of iterations increased, $T$ is reduced; hence, the details can be substantially protected.

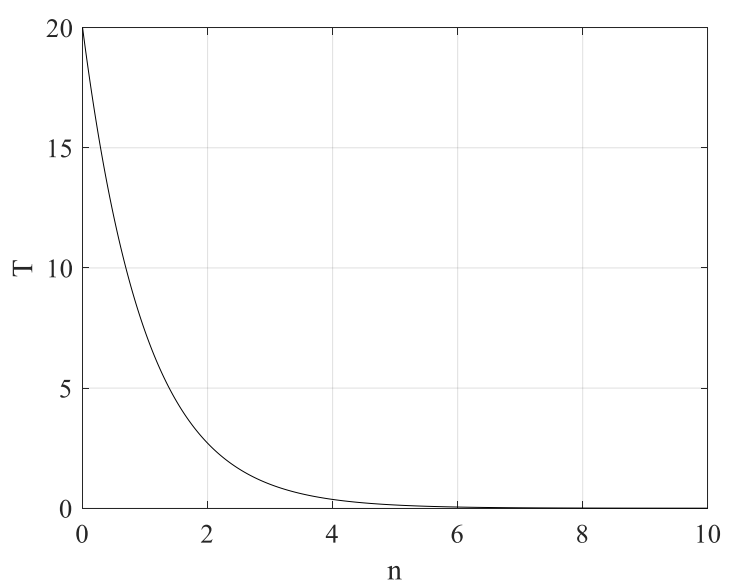

Fig. 4. Change Curve of $T$ as the Number of Iterations Increases when $T_{0}=20$

$\alpha\left(\sigma^{2}\right)$ and $\beta$ are used by the new algorithm model to control the normal and tangential diffusion coefficients. The enhancement idea is as follows.In the tangential direction, the edges and noise need forward diffusion and noise should be eliminated, thus, $\beta$ is a positive constant . In the normal direction, the local variance is within $0-T / 2$ and $\alpha$ is a positive value, the new model conducts forward diffusion and smoothen the noise. Within $T / 2-255, \alpha$ is a negative value. The new model conducts reverse diffusion to enhance the image features. A minimum value of -1 is obtained at the threshold value $T$; hence, the image details are substantially enhanced. As the local variance increases, $\alpha$ approaches 0 from -1 , the diffusion coefficient at the strong edges is reduced, the edge enhancement is prevented from becoming too fast, and overshoot occurs when the details are enhanced. Thereafter, the overshoot phenomenon appeares during the 
detail enhancement. Consequently, when the number $n$ of the iterations increases, the threshold value $T$ of the local variance is gradually reduced. $\alpha$ is decreased in the positive range. This way, the algorithm initially centeres on denoising; as the iterations proceeded, such algorithm eventually centeres on enhancement. The enhancement of the image details is guaranteed, while the noise is prevented from being enhanced.

\subsubsection{Numerical implementation}

Solving the process of $\frac{\partial^{2} f}{\partial n^{2}}$ and $\frac{\partial^{2} f}{\partial s^{2}}$ is as follows:

The derivative of the 2D grayscale image $f(x, y)$ for variables $x$ and $y$ is called the image gradient, which is expressed by $\nabla f$ as follows:

$$
\nabla f=\left(\frac{\partial f}{\partial x}, \frac{\partial f}{\partial y}\right)=\left(f_{x}, f_{y}\right)
$$

Thereafter, the image gradient modulus is defined as follows:

$$
|\nabla f|=\sqrt{f_{x}^{2}+f_{y}^{2}}
$$

The gradient direction of each point is as follows:

$$
n=\frac{\nabla f}{|\nabla f|}=\frac{\left(f_{x}, f_{y}\right)}{|\nabla f|}
$$

The directional derivative along the gradient is as follows:

$$
\frac{\partial f}{\partial n}=\nabla f \cdot n=\nabla f \frac{\nabla f}{|\nabla f|}=|\nabla f| \geq 0
$$

On the basis of the preceding equation (13):

$$
\begin{gathered}
\frac{\partial^{2} f}{\partial n^{2}}=\frac{\partial|\nabla f|}{\partial n}=\nabla(|\nabla f|) \cdot n \\
\text { If }|\nabla f|=\sqrt{f_{x}^{2}+f_{y}^{2}}, \text { then: }
\end{gathered}
$$

$$
\begin{aligned}
\nabla(\mid \nabla f) & =\nabla\left(\sqrt{f_{x}^{2}+f_{y}^{2}}\right)=\left(\frac{f_{x x} f_{x}+f_{x y} f_{y}}{\sqrt{f_{x}^{2}+f_{y}^{2}}}, \frac{f_{x y} f_{x}+f_{y y} f_{y}}{\sqrt{f_{x}^{2}+f_{y}^{2}}}\right) \\
& =\left(\frac{f_{x x} f_{x}+f_{x y} f_{y}}{|\nabla f|}, \frac{f_{x y} f_{x}+f_{y y} f_{y}}{|\nabla f|}\right) \\
\frac{\partial^{2} f}{\partial n^{2}} & =\left(\frac{f_{x x} f_{x}+f_{x y} f_{y}}{|\nabla f|}, \frac{f_{x y} f_{x}+f_{y y} f_{y}}{|\nabla f|}\right) \cdot \frac{\left(f_{x}, f_{y}\right)}{|\nabla f|} \\
& =\frac{f_{x x} f_{x}^{2}+2 f_{x y} f_{x} f_{y}+f_{y y} f_{y}^{2}}{f_{x}^{2}+f_{y}^{2}}
\end{aligned}
$$

In the $s=\frac{\left(-f_{y}, f_{x}\right)}{|\nabla f|}$ direction, the following equation can be obtained through a similar method:

$$
\frac{\partial^{2} f}{\partial s^{2}}=\frac{f_{x x} f_{y}^{2}-2 f_{x y} f_{x} f_{y}+f_{y y} f_{x}^{2}}{f_{x}^{2}+f_{y}^{2}}
$$

where $f_{x}, f_{y}, f_{x x}, f_{y y}$, and $f_{x y}$ of the image are calculated through the central difference scheme as follows:

$$
\left\{\begin{aligned}
f_{x} & =\frac{f_{i+1, j}-f_{i-1, j}}{2} \\
f_{y} & =\frac{f_{i, j+1}-f_{i, j-1}}{2} \\
f_{x x} & =f_{i+1, j}-2 f_{i, j}+f_{i-1, j} \\
f_{y y} & =f_{i, j+1}-2 f_{i, j}+f_{i, j-1} \\
f_{x y} & =\frac{f_{i-1, j-1}+f_{i+1, j+1}}{4}-\frac{f_{i-1, j+1}+f_{i+1, j-1}}{4}
\end{aligned}\right.
$$

\subsubsection{Algorithm steps}

This study uses the preceding principles and analysis as bases to conclude the implementation process of the proposed algorithm as follows:

(1) The image variance is calculated based on Equation(6), and the normalization processing of the local variance is conducted based on Equation(7).

(2) The initial value $T_{0}$ of the threshold value of the local variance is set. Equation(8)is used to calculate the control coefficient $\alpha\left(\sigma^{2}\right)$ in the normal direction.

(3) $\frac{\partial^{2} f}{\partial n^{2}}$ and $\frac{\partial^{2} f}{\partial s^{2}}$ are solved based on Equations(15)and (16).

(4) Equation (5) is used to process each point in the image.

(5) Steps (1) to (4) are repeated until a substantially denoised image $f$ is obtained.

\section{Result analysis and discussion}

The specific experimental verification was conducted in this section to evaluate the performance of the self-adaptive image enhancement algorithm based on the local variance. Moreover, the relevant study was conducted based mainly on the influences of the algorithm parameters on the processing effect and comparative study with other similar algorithms.

\subsection{Study of the main algorithm parameters}

The proposed algorithm involves the multiple parameters. The initial value $T_{0}$ of the threshold value of the local variance, the number $n$ of the iterations and the coefficient $\beta$ in the tangential direction on the experimental results was mainly analyzed in this section. During the specific experiment, other parameters were set as fixed values, and the processing effects when the different values were set for the parameters to be studied were compared to analyze the influence on algorithm performance. As the main objective depended on verifying the influences of the algorithm parameters, only the house image was used as the test image for the experimental data; the time step was $t=0.1$. 


\subsubsection{Influence of parameter $\beta$ in tangential direction}

To analyze the influence of coefficient $\beta$ in the tangential direction on enhancement effect, Fig.5 shows the result graphs of the algorithm that enhanced the house image under $\beta=0, \beta=0.5$, and $\beta=1$. The initial value of the threshold value was $T_{0}=8$ and the number of iterations was $n=4$. The result graphs show that the enhancement effects were different when different values were considered for $\beta$. When $\beta=0$, the image details could be enhanced but the image noise was also increased. When $\beta=1$, the noise and details were smoothed; when $\beta=0.5$, the details were enhanced, while noise was smoothed.

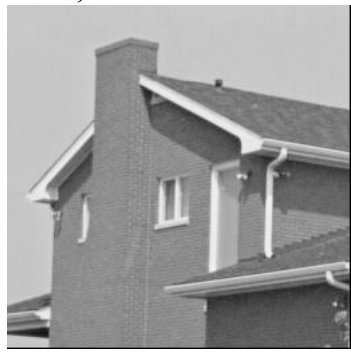

(a) Original House Image

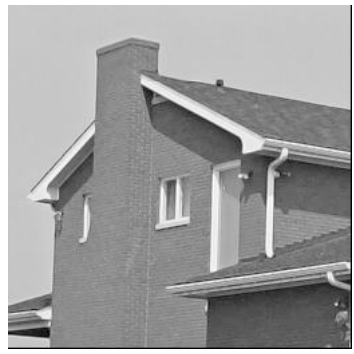

(c) $\beta=0.5$

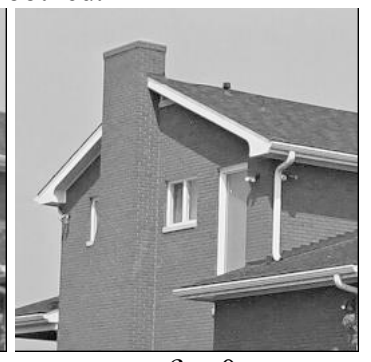

(b) $\beta=0$

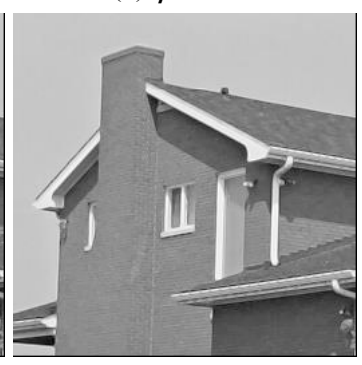

(d) $\beta=1$
Fig.5. Result Graphs of the House Image Enhancement under Different $\beta$

\subsubsection{Influence of the number $n$ of iterations}

To analyze the influences of the different parameters $n$ on the image enhancement effect, the house image was still used as the experimental image. The threshold initial value was $T_{0}=10$. Fig. 6 shows the result graphs of the house image enhancement under $n=2, n=5$, and $n=8$.

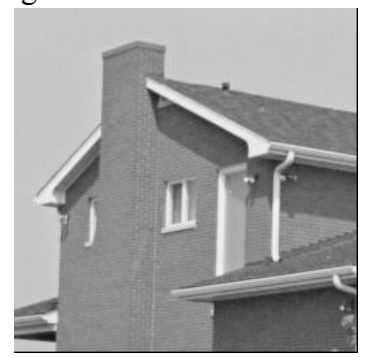

(a)Original House Image

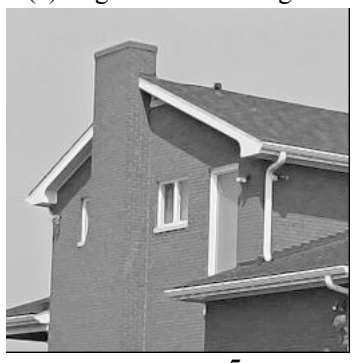

(c) $n=5$

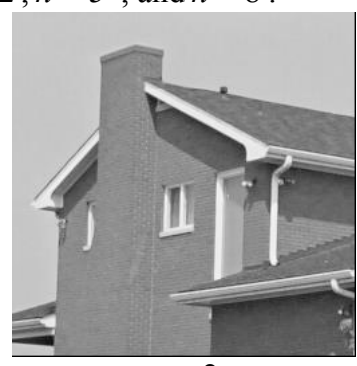

(b) $n=2$

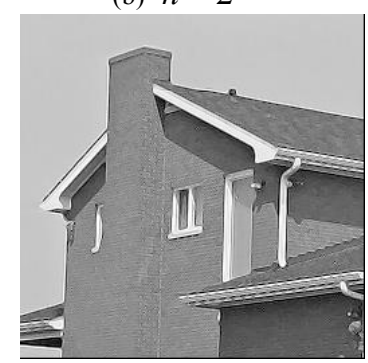

(d) $n=8$
Fig. 6. Result Graphs of the House Image Enhancement under Different Numbers of $n$ Iterations
Fig. 6 shows that when the number of iterations was small, the detail enhancement was weak. By contrast, as the number of iterations increased, the edges were gradually enhanced, but overshoot phenomenon appeared at the edges. To avoid the overshoot phenomenon of the edges, the number of iterations was generally selected as $n=5$.

\subsubsection{Influence of the initial value $T_{0}$ of threshold value}

To analyze the influence of the initial value $T_{0}$ of the threshold value on the enhancement result, the house image was analyzed and tested in the experiment. The number of iterations was set as $n=4 . T_{0}=10, T_{0}=30$, and $T_{0}=50$ were selected to enhance the image. An analysis of Fig. 7 indicated that when $T_{0}=10$, the edge details of the house were enhanced and the noise was amplified. When $T_{0}=50$, the background noise of the house was considerably inhibited, but the enhancement of the house edges, particularly eaves, was relatively weak. When $T_{0}=30$, background noise was inhibited while the edge details of the house were enhanced. Therefore, only when appropriate $T_{0}$ value was selected would the contradiction between the noise inhibition and the edge enhancement be solved.

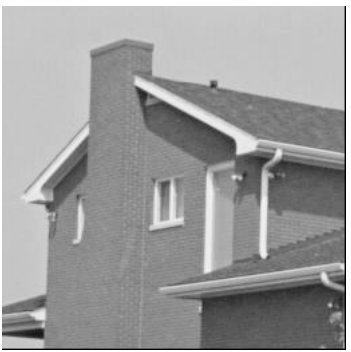

(a) Original House Image

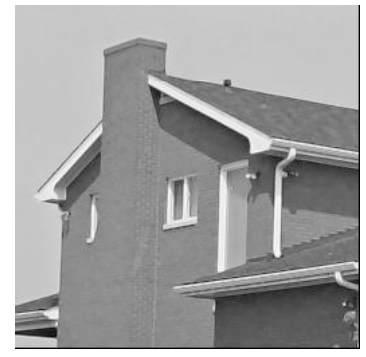

(c) $T_{0}=30$

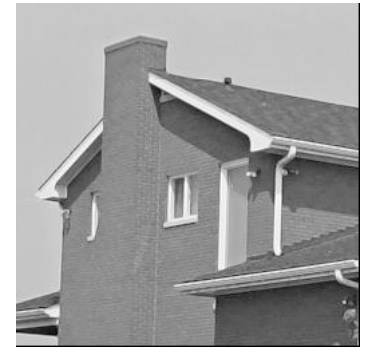

(b) $T_{0}=10$

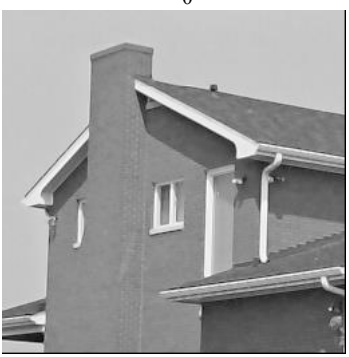

(d) $T_{0}=50$
Fig.7. Result Graphs of the House Image Enhancement under Different

\subsection{Comparative study with other algorithms}

The data used in the experiment were classical and lowcontrast images collected in an industrial site. To compare the algorithm in this chapter with similar algorithms, classical images and collected low-contrast images were selected in this section for algorithm test. In addition, a comparative study of the proposed algorithm with the traditional Laplace algorithm would be implemented. The relevant parameters were regulated in the experiment to make the processing results of all algorithms reach favorable visual effects.

The experimental results of the cameraman image are shown in Fig. 8. Accordingly, the traditional Laplace algorithm enhanced the details of the camera and hair in the image; however, evident burrs were generated in the overcoat sleeves and collar edges. However, the proposed algorithm enhanced the image details and effectively 
Yan Chen, Yunjiao Bai, Quan Zhang, Yanling Wang and Zhiguo Gui/

Journal of Engineering Science and Technology Review 10 (3) (2017) 64-72

inhibited the burr generation with the favorable visual effect. The traditional Laplace algorithm amplified the noise and enhanced the details with many noisy points in the image. The image enhanced by the proposed algorithm was relatively clear, and the noise enhancement was inhibited, thereby obtaining a substantial enhancement effect.

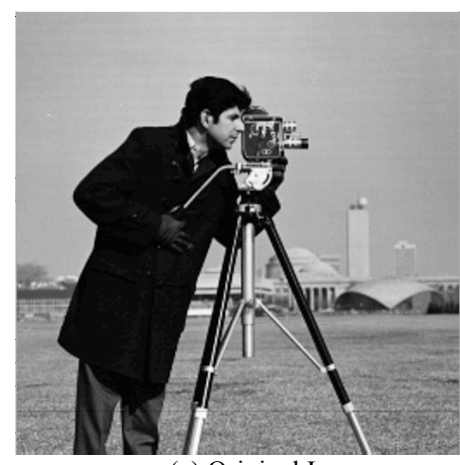

(a) Original Image

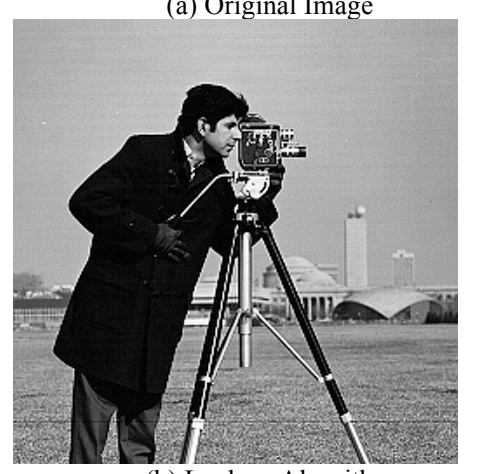

(b) Laplace Algorithm

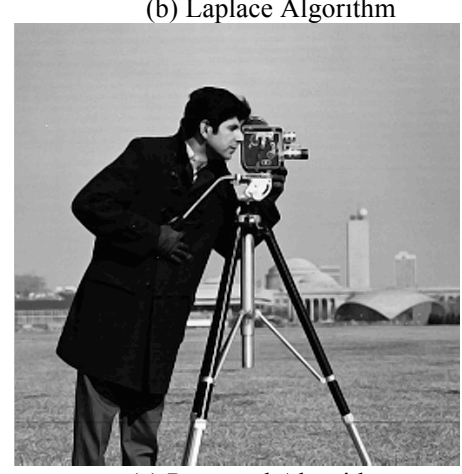

(c) Proposed Algorithm

Fig.8. Comparison of the Enhancement Effects of the Cameraman Image

To specify the enhancement effect of the new algorithm in a considerably objective manner, Table 1 lists the peak signal-to-noise ratios (SNR), the mean squared errors (MSE), and the information entropies of all enhanced images in Fig. 8. Table 1 shows that the image entropy values obtained through the proposed algorithm and the Laplace algorithm were identical. However, the image obtained through the proposed algorithm had high SNR and low MSE with the improved image quality. These results were consistent with those obtained through subjective observation.

Table 1. Objective Indexes of the Algorithms for the Cameraman Image

\begin{tabular}{c|c|c|c}
\hline & PSNR(db) & MSE & IE(bit) \\
\hline Laplace algorithm & 16.1803 & 39.5844 & 5.0452 \\
Proposed algorithm & 32.8720 & 5.7935 & 4.9023 \\
\hline
\end{tabular}

Fig. 9 shows the effect comparison of a baboon image enhancement using the traditional Laplace and proposed algorithms. The former enhanced the hair details in the image, although it evidently amplified the noise of the nose and eye corner details. In the image processed by the proposed algorithm, the baboon hairs were clear, the noise in the eye corners was evidently relieved, the noise was relatively smooth, and the visual effect was favorable.
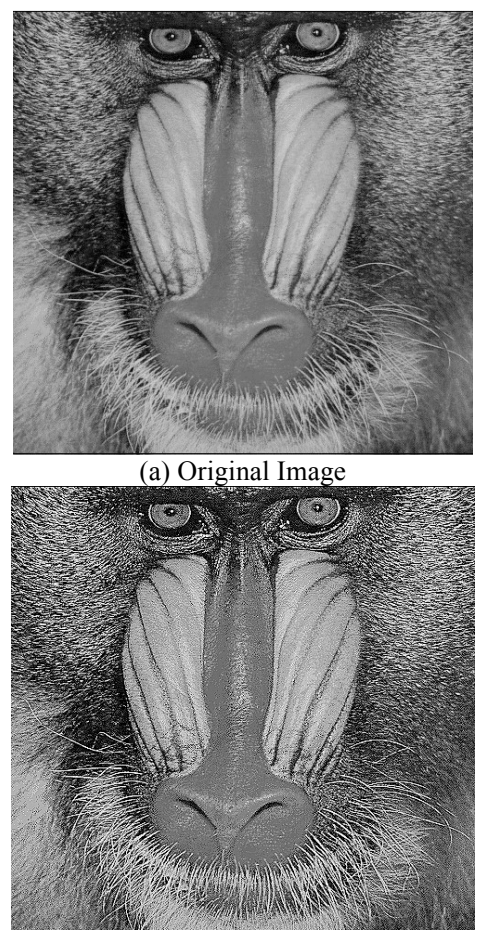

(b) Laplace Algorithm

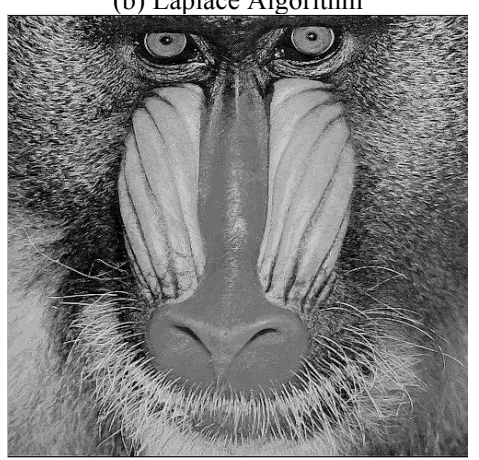

(c) Proposed Algorithm

Fig.9. Comparison of the Enhancement Effects of the Baboon Image

Table 2 lists the objective indexes of the images obtained after processing using the two algorithms. The image processed by the proposed algorithm had high peak SNR with low MSE. These results were consistent with subjective observation results. The image enhanced by the proposed algorithm had extensive detailed information and its noise was inhibited.

Table 2. Objective Indexes of Algorithms for Baboon

\begin{tabular}{|c|c|c|c|}
\hline & PSNR(db) & MSE & IE(bit) \\
\hline Laplace algorithm & 3.9880 & 161.1171 & 4.2012 \\
\hline Proposed algorithm & 22.0618 & 20.1118 & 5.2885 \\
\hline
\end{tabular}

Fig. 10 shows the effect comparison of the low-contrast industrial image collected on the site and processed through the traditional Laplace enhancement and the proposed algorithms. The parameters include the initial value of the threshold value of $T_{0}=1$ and the number of iterations was $n=5$. Fig. 10 shows (a) the industrial image, (b) the image 
enhanced using the Laplace algorithm and (c), the image enhanced by the proposed algorithm.
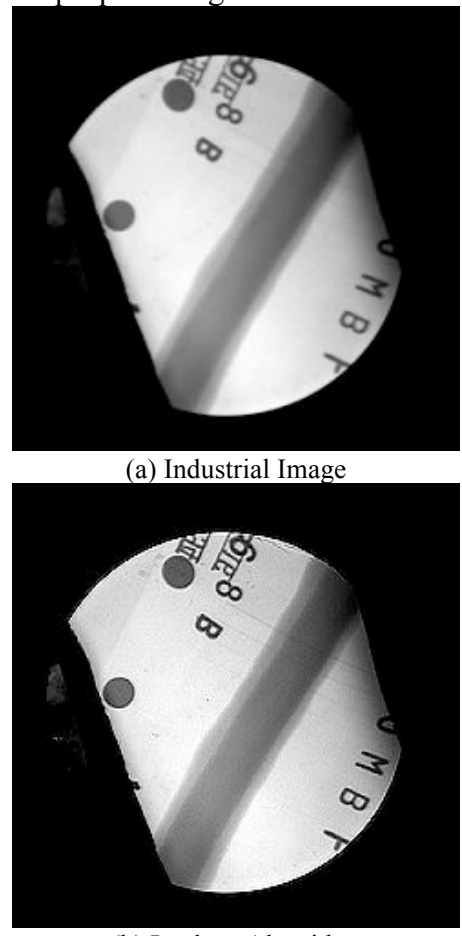

(b) Laplace Algorithm

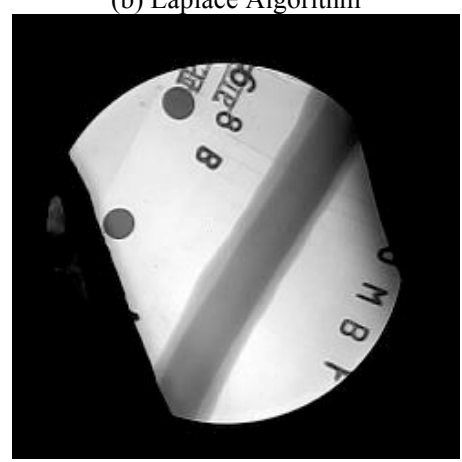

(c) Proposed Algorithm

Fig. 10. Comparison of the Enhancement Effects of the Industrial Image

Table 3 provides the objective evaluation indexes of the lowcontrast industrial image after processing using the different methods. To intuitively compare the objective indexes of the different algorithms, Fig. 11 shows the histograms of the objective indexes of the low-contrast image enhancement. The Laplace algorithm was sensitive to noise; hence, it had high information entropy. However, the image processed by the proposed algorithm had high peak SNR and low MSE, thereby sufficiently certifying the advantages of the proposed algorithm.

Table 3. Objective of the Indexes of the Algorithms for the Industrial Image

\begin{tabular}{|c|c|c|c|}
\hline & PSNR(db) & MSE & IE(bit) \\
\hline Laplace algorithm & 23.8584 & 16.3539 & 3.1213 \\
\hline Proposed algorithm & 30.8252 & 7.3330 & 3.1121 \\
\hline
\end{tabular}

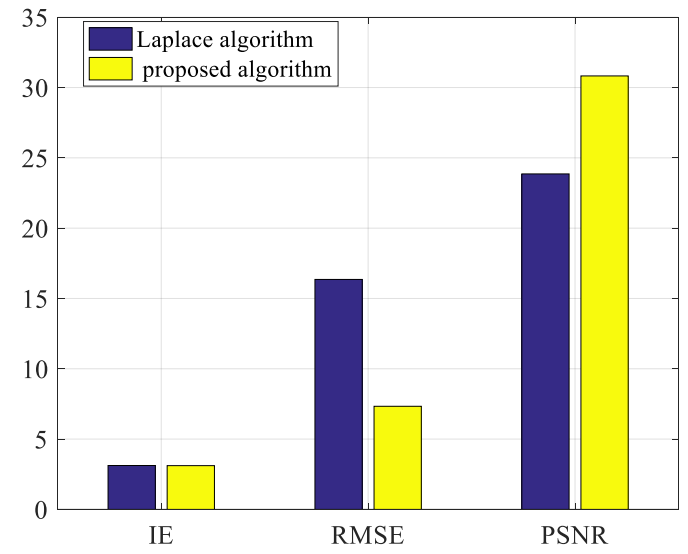

Fig.11. Histograms of the Objective Indexes of the Industrial Image

\section{Conclusions}

To solve the problem in which the traditional Laplace algorithm amplified noise and enhanced image details, the local variance was adopted to classify image features, the change characteristics of the trigonometric function were used to construct the diffusion coefficients, an anisotropic image enhancement method was proposed, and the influences of the multiple parameters on image enhancement were analyzed through simulation experiment. The conclusions obtained are as follows.

(1)The image enhancement algorithm model established based on the local variance can accurately calculate the local neighborhood features of the pixels and reflect the change features of the image details during the pixel diffusion process.

(2)The comparison of the enhancement effects between the proposed algorithm and traditional Laplace algorithm indicates that the anisotropic diffusion method can differentiate image noise and details. In addition, the organic combination of the self-adaptive threshold value and the diffusion can solve the problem of sensitivity of diffusion to noise.

(3) The simulation analysis of the low-contract image enhancement algorithm shows that low-contrast image detail information is substantially protected by regulating the number of iterations of tangential direction parameters. In addition, the peak SNR reaches 30.8252 , MSE is as low as 7.333 , information entropy remains unchanged, the expected effect is obtained, the threshold value parameter is reasonable, and the enhancement effect of the improved algorithm is stable.

The neighborhood features in the proposed algorithm can and accurately reflect the local features of the pixels in consideration of the local variances of the pixel. This process aims to provide a substantially accurate technical support for the image enhancement method based on partial differential equation. However, the parameter setting of the enhancement model needs regulation by experience. Thus, a man-machine interaction mode for parameter setting should be established.

This is an Open Access article distributed under the terms of the Creative Commons Attribution Licence

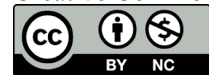




\section{References}

1.Han X. Z., Zhao J., "Image Texture and Contrast Enhancement through Combination of Partial Differential Equation". Optical Precision Engineering, 20(6), 2012,pp.1382-1388.

2.Zhang H., Chen H. M., "Image Edge Detection and Filtering Processing Technology Based on Fusion of SUSAN Algorithm and Robert Algorithm". Computer Science, 40(3), 2013,pp.302-304.

3.Das,S.,"Comparison of various edge detection technique". International Journal of Signal Processing, Image Processing and Pattern Recognition, 9(2), 2016,pp.143-158.

4.Wu J, Chen D.Z., Guo C.Z., "Image Enhancement Realized through Variation of Sobel Edge Detection Algorithm". Laser and IR, 38(6), 2008,pp.612-614.

5.Sun Z. G., Han C. Z., "Image Enhancement based on Laplacian Operator". Computer Application Research, 24(1), 2007,pp.222-223.

6. Mallick A, Roy S, Chaudhuri S. S., et al, "Optimization of Laplace of Gaussian (LoG) filter for enhanced edge detection: A new approach". International Conference on Control, Instrumentation, Energy and Communication, Kolkata, India: IEEE, 2014,pp.658-661.

7.Han D. Y., "Histogram Equalization Method of Detail Reservation of Low-contrast Video Image". Computer Simulation, 30(8), 2013,pp.233-236.

8. Zhou Z. G., Sang N., Hu X. R,, "Global brightness and local contrast adaptive enhancement for low illumination color image“. Optik, 125(2014), 2013,pp.1795-1799.

9.Xiao J. S., Shan S. S., Duan P. F.,et al., "Rapid Image Enhancement Algorithm based on Fusion of Different Color Spaces". Journal of Automation, 40(4), 2014, pp.697-705.
10.Zhao, X. F., "Nonlinear diffusion filtering method based on wavelet image". International Journal of Multimedia and Ubiquitous Engineering,9(9),2014,pp.29-40.

11.Perona P,Malik J., "Scale-Space and Edge Detection Using Anisotropic Diffusion". IEEE Transaction on Patteron Analysis and Machine Intelligence.12(7), 1990,pp.629-639.

12. Catté F., Lions P. L., Morel J. M., et al., "Image selective smoothing and edge detection by nonlinear diffusion". SIAM Journal on Numerical analysis, 29(1), 1992,pp.182-193.

13. Guo Z., Sun J., Zhang D., et al., "Adaptive Perona-Malik model based on the variable exponent for image denoising". IEEE Transactions on Image Processing, 21(3), 2012,pp. 958-967.

14. Ratner V., Zeevi Y. Y., "Denoising-enhancing images on elastic manifolds". IEEE Transactions on Image Processing, 20(8), 2011,pp. 2099-2109.

15. You Y. L., Kaveh M., "Fourth-order partial differential equations for noise removal". IEEE Transactions on Image Processing, 9(10), 2000,pp.1723-1730.

16.Bai J., Feng X. C., "Fractional-order anisotropic diffusion for image denoising". IEEE Transactions on Image Processing,16(10), 2007,pp.2492-2502.

17. Wang Y. H., Liu W. N., Chen A.H., et al., "Nonlinear dim target enhancement algorithm based on partial differential equation". Journal of Dalian Maritime University,34(2), 2008. pp.57-60.

18. Chao S. M., Tsai D. M., “An improved anisotropic diffusion model for detail-and edge-preserving smoothing". Pattern Recognition Letters, 31(13), 2010, pp.2012-2023. 